\title{
0 processo de avaliação em ciência e a indicação de Carlos Chagas ao prêmio Nobel de Fisiologia ou Medicina
}

\author{
The assessment process within science and the nomination \\ of Carlos Chagas for the Nobel prize for Physiology or Medicine
}

\author{
José Eymard Homem Pittella ${ }^{1}$
}

\begin{abstract}
RESUMO
Uma das maiores realizações na história da medicina foi a descrição da doença de Chagas pelo médico e cientista Carlos Chagas. Ao completar 100 anos da descoberta da doença de Chagas, permanecem ainda especulações a respeito das duas indicações oficiais de Carlos Chagas à maior premiação mundial em ciência, o Nobel, em 1913 e 1921. Admite-se que a não premiação do genial cientista possa ter ocorrido em razão da forte oposição que enfrentou no Brasil por parte de alguns médicos e pesquisadores da época, que chegaram mesmo a questionar a existência da doença de Chagas, influenciando a decisão do Comitê Nobel para não premiá-lo. A análise do banco de dados dos arquivos do Prêmio Nobel, com a revelação dos nomes de indicadores, indicados e ganhadores do prêmio, cobrindo o período 1901-1951, trouxe informações não apenas sobre o que era considerada realização científica na época, mas também sobre quem eram os cientistas importantes e quais eram as relações entre eles. 0 não reconhecimento das descobertas de Carlos Chagas pelo Comitê Nobel parece ser mais corretamente explicado por esses fatores do que pelo impacto negativo da oposição local.
\end{abstract}

Palavras-chaves: Carlos Chagas. Doença de Chagas. Prêmio Nobel. Medicina. História.

\begin{abstract}
One of the greatest achievements in the history of medicine was the description of Chagas disease by the physician and scientist Carlos Chagas. A hundred years after the discovery of the disease, speculation still remains regarding the two official nominations of Carlos Chagas for the Nobel Prize, the biggest worldwide scientific award, in 1913 and in 1921. It has been accepted that the reason why the prize was not awarded to this brilliant scientist may have been the strong opposition that he faced in Brazil, from some physicians and researchers of that time. They went as far as questioning the existence of Chagas disease, thereby possibly influencing the decision of the Nobel Committee not to award the prize to him. Analysis of the database of the Nobel prize archives, with the revelation of the names of nominators, nominees and prizewinners spanning the years 1901-1951, brought information not only about what was considered to be a scientific achievement at that time, but also about who the important scientists were and what the relationships between them were. The non-recognition of Carlos Chagas' discoveries by the Nobel Committee appears to be more correctly explained by these factors than by the negative impact of the local opposition.
\end{abstract}

Key-words: Carlos Chagas. Chagas disease. Nobel Prize. Medicine. History.

Ao completar 100 anos da descoberta da doença de Chagas, permanecem ainda incertezas e especulações a respeito da história da indicação de Carlos Chagas (1879-1934) à maior premiação mundial em ciência, o Nobel. Os artigos de Coutinho ${ }^{13}$, Coutinho e cols ${ }^{14}$, Dias ${ }^{16}$ e Dias e cols ${ }^{17}$, publicados por ocasião dos 90 anos da descoberta da doença de Chagas, são praticamente os únicos que abordam a questão e comentam aspectos das duas indicações oficiais de Carlos Chagas ao prêmio Nobel de Fisiologia ou Medicina, em 1913 e 1921. Na realidade, pode-se dizer que esses quatro artigos são complementares, pois dois

1. Departamento de Anatomia Patológica e Medicina Legal, Faculdade de Medicina, Universidade Federal de Minas Gerais, Belo Horizonte,MG.

Endereço para correspondência: Dr. José Eymard Homem Pittella. Rua dos Otoni 712 Conj 304, Bairro Santa Efigênia, 30150-270 Belo Horizonte, MG

e-mail: pittella@medicina.ufmg.br

Recebido para publicação em 05/12/2008

Aceito em 29/01/2009 dos quatro autores são os mesmos, isoladamente ou em dupla, nos quatro textos. Para os autores, o pouco tempo decorrido entre a publicação e a divulgação da nova entidade, além do fato de que no período 1909-1912 somente 14 trabalhos haviam sido publicados sobre a doença de Chagas, sendo 12 de autoria do próprio Carlos Chagas, talvez tenha sido o responsável pela sua não premiação em 1913. Em relação à premiação de 1921, os autores argumentam que um dos motivos de ele não ter ganhado o prêmio Nobel foi a forte oposição que enfrentou no Brasil, particularmente em Manguinhos e na Academia Nacional de Medicina, por parte de alguns médicos e pesquisadores da época, seja por inveja ou incompetência, que chegaram mesmo a questionar a existência da doença de Chagas. 0 historiador argentino Sierra-Iglesias especula que essa forte oposição tenha influenciado o Comitê Nobel para não premiá-lo nesse ano ${ }^{25}$. E o que seria mais estranho: em 1921 não houve prêmio Nobel para a área de Fisiologia ou Medicina e que Carlos Chagas teria sido o único cientista indicado à premiação nesse ano. Este texto 
tem, portanto, o objetivo de esclarecer essas questões, situando a indicação de Carlos Chagas ao prêmio Nobel dentro do contexto da avaliação da atividade científica em geral e dos fatores culturais e históricos que influenciavam a escolha dos indicadores (pessoas qualificadas a indicar alguém para o prêmio) e indicados ao prêmio. 0 artigo será assim dividido em duas partes: 1) Aspectos históricos do processo de indicação ao prêmio Nobel de Fisiologia ou Medicina; 2) A indicação de Carlos Chagas ao prêmio Nobel de Fisiologia ou Medicina.

\section{ASPECTOS HISTÓRICOS DO PROCESSO DE INDICAÇÃO AO PRÊMIO NOBEL DE FISIOLOGIA OU MEDICINA}

A análise de alguns aspectos da maior premiação mundial em ciência, o Nobel, é altamente esclarecedora do papel que os fatores humanos, culturais e históricos têm sobre a avaliação da atividade científica. 0 processo da premiação se inicia com a indicação de candidatos ao prêmio por pessoas qualificadas para tal $^{26}$. Os candidatos indicados por pessoas qualificadas são selecionados preliminarmente pelo Comitê Nobel, o qual é constituído de cinco membros e o secretário da Assembléia Nobel $^{20}$. Os candidatos selecionados preliminarmente são então submetidos à avaliação por especialistas indicados pelo Comitê Nobel, quais sejam, membros da Assembléia Nobel e revisores externos. Com base nessa avaliação o Comitê Nobel elabora um relatório recomendando uma lista de candidatos para a avaliação final pela Assembléia Nobel no Instituto Karolinska, sede da Faculdade de Medicina. A Assembléia possui 50 membros e a escolha do premiado é feita por votação majoritária. A decisão é final e sem apelação. As informações sobre quem são os indicadores e indicados permanecem secretas durante 50 anos. 0 prêmio será concedido às pessoas responsáveis pela descoberta, invenção ou aperfeiçoamento que fosse de benefício para a humanidade. Somente podem ser indicados ao Nobel os nomes que forem indicados por pessoas devidamente qualificadas. 0 Comitê Nobel recebe muitas indicações feitas por pessoas não qualificadas para tal, e essas indicações não são incluídas entre os documentos examinados pelo Comitê Nobel. Não pode haver auto-indicação e nem de pessoas já falecidas.

Desde o início, a premiação atraiu grande interesse, não somente tendo em vista a importância da ciência e suas aplicações na vida das pessoas, percebidas pela sociedade da época, mas também pela novidade de se fazer uma grande doação para causas científicas ou filantrópicas. A partir da instituição da premiação os estatutos da Fundação Nobel estipulavam que as atas de deliberação do Comitê Nobel seriam mantidas secretas e que as decisões de premiação não poderiam ser contestadas. Essas regras foram originalmente estabelecidas com o intuito de proteger o prestígio da premiação contra a publicidade adversa que poderia resultar no caso de algum não premiado descontente acessar as atas e apelar da decisão. Com o passar dos anos e com a crescente fama do prêmio Nobel, o sigilo que envolve a escolha da premiação provou também ser de utilidade para proteger as pessoas encarregadas da difícil tarefa de selecionar os laureados do prêmio. A abertura, em 1974, dos arquivos do prêmio Nobel na Academia Real de Ciências Sueca e a divulgação pela Internet, a partir de 1995, do banco de dados de indicadores e indicados ao prêmio (www.nobel.se), permitiu que documentos datados de pelo menos 50 anos fossem analisados por pesquisadores.

A análise desses documentos, com a revelação dos nomes de ganhadores e perdedores do prêmio na primeira metade do século $\mathrm{XX}$, trouxe informações sobre o que era considerada realização científica na época, quem eram os cientistas importantes e quais eram as relações entre eles ${ }^{15}$. A avaliação dos critérios para a indicação de um ou outro candidato ao prêmio mostrou o papel importante desempenhado pelas próprias idéias que os membros do Comitê Nobel tinham sobre o tipo de trabalho científico que poderia ser premiado. Nesse sentido os membros do Comitê Nobel eram guiados tanto por seus próprios interesses em ciência quanto por decisões anteriores de premiação. As ligações dos membros do Comitê Nobel com a comunidade científica internacional, centrada quase que exclusivamente em cientistas europeus e norte-americanos, influenciavam também nas suas escolhas.

A divulgação do censo de indicadores e indicados ao Prêmio Nobel de Fisiologia ou Medicina no período 1901-1951 ${ }^{1}$ (Tabela 1) mostra de fato que a grande maioria provinha dos Estados Unidos e países da Europa Ocidental, havendo certa relação entre número de indicadores, de indicados e de premiados do mesmo país (as exceções são a Itália, Polônia e Japão). De fato, quando se examina detalhadamente quem era quem entre os

\section{TABELA 1}

Banco de dados de indicadores, indicados e premiados para o prêmio Nobel de Fisiologia ou Medicina, no período 1901-1951, distribuídos por países (apenas os 20 mais freqüentes)*, além do Brasil ${ }^{* * *}$

\begin{tabular}{|c|c|c|c|}
\hline Países & Indicadores & Indicados & Premiados \\
\hline Estados Unidos & 732 & 1.132 & 14 \\
\hline França & 755 & 930 & 4 \\
\hline Alemanha & 763 & 914 & 9 \\
\hline Reino Unido & 256 & 464 & 9 \\
\hline Itália & 312 & 253 & 1 \\
\hline Suécia & 174 & 167 & 1 \\
\hline Bélgica & 240 & 153 & 2 \\
\hline Áustria & 157 & 150 & 2 \\
\hline Polônia & 140 & 140 & 0 \\
\hline Suíça & 168 & 129 & 4 \\
\hline Japão & 105 & 98 & 0 \\
\hline Holanda & 82 & 97 & 2 \\
\hline Rússia & 118 & 82 & 2 \\
\hline Dinamarca & 63 & 68 & 4 \\
\hline Espanha & 59 & 56 & 1 \\
\hline Canadá & 40 & 49 & 1 \\
\hline Argentina & 28 & 49 & 1 \\
\hline Hungria & 75 & 41 & 1 \\
\hline República Tcheca & 62 & 29 & 0 \\
\hline Portugal & 21 & 23 & 1 \\
\hline Brasil & 24 & 4 & 0 \\
\hline
\end{tabular}

*http://nobelprize.org/nomination/medicine/country.htm

**http://nobelprize.org/nomination/medicine/nomination.php?action=advsearch\& key $1=$ candcountry\&log 1 
indicadores e indicados, vê-se que era comum a indicação de compatriotas. Nota-se, ainda, em alguns casos, contraste entre 0 número de indicações e o de premiações recebidas. Basta lembrar que Frederick G. Banting (1841-1941) e John J. R. Macleod (18761935) receberam o prêmio Nobel de Fisiologia ou Medicina de 1923 pela descoberta da insulina tendo recebido apenas três indicações naquele mesmo ano, enquanto que Robert Koch (1843-1910) recebeu o prêmio Nobel de Fisiologia ou Medicina de 1905 por sua descoberta do bacilo causador da tuberculose, somente após ter sido indicado 55 vezes durante quatro anos consecutivos. E alguns cientistas foram campeões de indicações, sem jamais terem recebido a premiação, como p. ex., os físicos Arnold Sommerfeld (indicado 74 vezes), Vilhelm Bjerknes (54 vezes) e Friedrich Paschen ( 45 vezes), o químico Gilbert Newton Lewis ( 42 vezes) e os médicos Ferdinand Sauerbruch ( 54 vezes) e Sigmund Freud (32 vezes). Quatro cientistas brasileiros foram indicados ao Nobel de Fisiologia ou Medicina no período 19011951: Carlos Chagas (em 1913 e 1921); Antônio Cardoso Fontes (1879-1943), em 1934, por seu trabalho sobre o bacilo da tuberculose; Adolfo Lutz (1855-1940), em 1938, por seu estudo sobre algumas doenças tropicais; Manoel de Abreu (1894-1962), em 1946, pela introdução da abreugrafia.

\section{A INDICAÇÃO DE CARLOS CHAGAS AO PRÊMIO NOBEL DE FISIOLOGIA OU MEDICINA}

Uma das maiores realizações na história da medicina, sem precedentes na investigação científica, foi a descrição da doença de Chagas (também conhecida como tripanossomíase americana) pelo médico e cientista Carlos Chagas. De fato, ao participar de uma campanha de prevenção e tratamento da malária no norte de Minas Gerais, entre 1907 e 1909, Carlos Chagas identificou o agente etiológico da nova doença (o Trypanosoma cruzi), o ciclo evolutivo do Trypanosoma cruzi, o inseto transmissor do Trypanosoma cruzi (o barbeiro, hemíptero hematófago da subfamília Triatominae), o reservatório silvestre do Trypanosoma cruzi (sagui, tatu-galinha) e, posteriormente, observou o Trypanosoma cruzi no sangue de indivíduos acometidos, descrevendo as duas modalidades clínicas de apresentação da doença (as formas aguda e crônica) e as suas manifestações clínicas. Em relação ao acometimento do coração pelo Trypanosoma cruzi (forma crônica cardíaca), descreveu os distúrbios do ritmo cardíaco como os elementos mais marcantes do quadro clínico, ao lado da insuficiência cardíaca, identificando os bloqueios de condução (acometimento do sistema excito-condutor do coração). Além disso, Carlos Chagas reproduziu a doença inoculando o Trypanosoma cruzi em animais de laboratório, como cobaias e sagüis.

A sequiência de descobertas conduzida por Carlos Chagas é inédita na história das doenças infecciosas e parasitárias por dois motivos: 1) identificou em cerca de dois anos de trabalho uma nova doença, o agente etiológico e seu ciclo evolutivo, o modo de transmissão, o reservatório natural do parasita e reproduziu experimentalmente a doença em animais; 2) contrariamente ao que ocorre na história das doenças infecciosas e parasitárias, na qual a descrição da doença antecede a identificação de seu agente etiológico, do modo de transmissão e dos reservatórios naturais, ele seguiu um caminho inverso, partindo do inseto transmissor infectado para a doença por ele provocada.

Dois exemplos de que a descrição de uma doença infecciosa antecede a identificação de sua causa são a tuberculose e a síndrome da imunodeficiência adquirida (AIDS/SIDA). A tuberculose era uma doença conhecida na Europa desde o início da Idade Média, tendo sido a primeira doença infecciosa a ser reconhecida como tal, ou seja, transmissível de pessoa para pessoa, pelo médico, matemático, geógrafo, astrônomo e poeta Girolamo Fracastoro (1478-1553) em 1546, uma idéia revolucionária na época ${ }^{24}$. Entretanto, somente em 18920 médico e cientista Robert Koch identificou o bacilo causador da doença, o Mycobacterium tuberculosis. Conforme mencionado nos parágrafos precedentes, por essa descoberta Koch ganhou o prêmio Nobel de Fisiologia ou Medicina de 1905. Os primeiros casos de AIDS foram descritos nos EUA em 1981 e a natureza infecciosa da doença, suspeitada praticamente desde o início, foi confirmada em 1983 com o isolamento do HIV por pesquisadores do Instituto Pasteur ${ }^{2}$. Posteriormente, o vírus foi denominado HIV-1 com a descoberta, em 1986, de outra cepa de HIV, o HIV-2, este último quase que restrito à África Ocidental ${ }^{12}$. A descoberta do HIV rendeu aos cientistas Françoise Barré-Sinoussi e Luc Montagnier o prêmio Nobel de Fisiologia ou Medicina de 2008.

As descobertas de Carlos Chagas chegaram ao conhecimento da comunidade científica internacional da época, em parte facilitado pela publicação de seu principal trabalho na Revista Memórias do Instituto Oswaldo Cruz, em $1911^{10}$, simultaneamente nos idiomas português e alemão (esse último, juntamente com o inglês e francês, era um dos idiomas de divulgação científica da época). Carlos Chagas já havia publicado em 1909, na mesma revista, os achados sobre a morfologia e o ciclo evolutivo do Trypanosoma cruzi, o agente etiológico da nova doença ${ }^{7}$. Nos anos 1909-1913, ele publicou algumas de suas observações em revistas francesas e alemãs $5689{ }^{11}$. Apesar da sequiência de descobertas extraordinárias em um curto período de tempo e da publicação de seus principais achados em revistas e idiomas internacionais, o genial cientista brasileiro não foi contemplado com a premiação máxima, o prêmio Nobel. Na época houve pouco interesse dos pesquisadores europeus e norte-americanos sobre a descoberta de uma nova doença restrita à America Latina. No final do século XIX e início do século XX, período que corresponde ao apogeu da expansão imperialista mundial das potências européias, a atenção dos europeus estava voltada para suas colônias na Ásia e África e para as doenças tropicais que acometiam os nativos destes continentes e que podiam afetar também os colonizadores e, assim, prejudicar a expansão do colonialismo europeu naqueles continentes $^{1421}$. Foi essa preocupação que levou, nessa mesma época, ao surgimento dos institutos científicos europeus voltados para o estudo das doenças tropicais: Instituto Pasteur em 1887, em Paris; Liverpool School of Tropical Medicine em 1898, em Liverpool; London School of Hygiene \& Tropical Medicine em 1899, em Londres; Institut für Tropenmedizin em 1900, em Hamburgo.

Carlos Chagas recebeu somente duas indicações oficiais ao prêmio Nobel, ambas de professores universitários brasileiros. 
Outras duas indicações não oficiais foram feitas, mas sem registro pelo Comitê Nobel. A primeira indicação oficial, em 1913, foi feita por Manuel Augusto Pirajá da Silva (1873-1961), professor de parasitologia na Faculdade de Medicina da Universidade da Bahia. Pirajá da Silva ficou conhecido por ter sido o primeiro pesquisador a identificar o verme adulto do Schistosoma mansoni, em 1908. Pirajá da Silva fizera cursos na Europa, se relacionava e era respeitado pelos seus colegas europeus ${ }^{14}$. Pirajá da Silva conhecia os trabalhos de Carlos Chagas e escreveu um texto bem fundamentado sobre a descoberta da doença de Chagas. Em 1913, 118 pessoas qualificadas para tal indicaram 63 cientistas ao prêmio Nobel de Fisiologia ou Medicina ${ }^{1}$. Dos 63 indicados, apenas 10 tiveram seus nomes avaliados e recomendados por membros do Comitê Nobel ou para tal designados (Carl Sundberg, JG Edgreen, JE Johansson, J Äkerman e KA Mömer). Charles Richet (1850-1935), com nove avaliações e recomendações, foi o ganhador do prêmio daquele ano, por suas contribuições ao estudo da anafilaxia (Richet já havia recebido oito indicações no período 1904-1912). Carlo Forlanini (1847-1918), com oito avaliações e recomendações, foi o segundo colocado, por ter utilizado o pneumotórax artificial no tratamento da tuberculose pulmonar. A única indicação obtida por Carlos Chagas, efetuada por Pirajá da Silva, não foi avaliada e recomendada, mostrando que a doença descoberta por ele, apesar de todo a sua genialidade e do ineditismo de sua descoberta na história das doenças infecciosas e parasitárias, não havia causado o merecido impacto na comunidade científica internacional. 0 fato de que até 1912 somente 14 trabalhos haviam sido publicados sobre a doença de Chagas, 12 dos quais do próprio Carlos Chagas, mostra 0 escasso envolvimento de outros pesquisadores no estudo da nova entidade, contribuindo para restringir a sua divulgação entre a comunidade científica ${ }^{17}$.

A segunda indicação oficial de Carlos Chagas ao prêmio Nobel foi feita em 1921 por Hilário Soares de Gouvêa (18431923) professor de oftalmologia e otorrinolaringologia na Faculdade de Medicina da Universidade do Brasil (atual UFRJ). Hilário de Gouvêa foi membro titular da Academia Nacional de Medicina e participou de diversos congressos internacionais de medicina na Europa, tendo apresentado e publicado vários trabalhos em congressos e revistas nacionais e internacionais ${ }^{18}$. Ele escreveu uma carta mostrando o talento, reputação, altruísmo e dedicação de Carlos Chagas à ciência, descrevendo resumidamente a seqüência de descobertas sobre o agente transmissor, o tripanossomo, o modo de transmissão e as repercussões clínicas e anátomo-patológicas graves da doença. 0 documento foi acompanhado de uma brochura sobre o Instituto Oswaldo Cruz, de oito trabalhos originais de Carlos Chagas e do texto de uma conferência de Oswaldo Cruz sobre algumas doenças protozoárias ${ }^{14}$. Uma exposição detalhada das razões da candidatura de Carlos Chagas foi também enviada, provavelmente representando o documento anônimo de oito páginas arquivado na biblioteca do Instituto Oswaldo $\mathrm{Cruz}^{3}$.

Entre a primeira e a segunda indicação Carlos Chagas ganhara também maior reconhecimento nacional e internacional ${ }^{14}$. A indicação de Carlos Chagas mais uma vez não recebeu avaliação e recomendação pelo Comitê Nobel. Segundo Nils Ringertz, secretário do Comitê Nobel para Fisiologia ou Medicina, em carta enviada para a pesquisadora da Universidade de São Paulo, Marília Coutinho, a indicação foi examinada por Gunnar Hedrén, um dos membros do Comitê Nobel, que fez um relatório oral aos demais membros do Comitê $\hat{e}^{13}$. Em 1921, 82 pessoas qualificadas para tal indicaram 42 cientistas ao prêmio Nobel de Fisiologia ou Medicina ${ }^{1}$. Eugène Gley (1857-1930), por suas descobertas sobre as funções da paratireóide e tireóide, Émile Roux (1853-1933), cofundador do Instituto Pasteur e descobridor do soro anti-diftérico, Henri Duret (1849-1921), por seus trabalhos sobre a fisiologia e patologia cerebral e Charles Sherrington (1857-1952), pelos estudos sobre a função do neurônio foram os que receberam mais indicações (11, nove, sete e sete, respectivamente). No entanto, as indicações dos dois primeiros não receberam avaliação e recomendação, enquanto que as de Duret e Sherrington foram avaliadas e recomendadas por membros do Comitê Nobel ou para tal designados (J Äkerman, JE Johansson, Alfred Pettersson). 0 fato é que não houve concessão do prêmio Nobel de Fisiologia ou Medicina no ano de 1921, embora tenham sido concedidos os de Física para Albert Einstein (1879-1955) ${ }^{29}$ e o de Química para Frederick Soddy (1877-1956) ${ }^{27}$. Sherrington ganharia 0 prêmio Nobel de $1932^{28}$.

Não se sabe ao certo as razões para a não concessão da premiação do Nobel em Fisiologia ou Medicina em 1921, a qual também não foi concedida em $1925^{28}$, embora tenha sido concedida a de Física para James Franck (1882-1964) e Gustav Hertz (1887-1975) ${ }^{29}$ e a de Química para Richard Zsigmondy $(1865-1929)^{27}$. E mais uma vez, não foi por falta de indicações. Para o prêmio Nobel de Fisiologia ou Medicina de 1925, 127 pessoas qualificadas para tal indicaram 66 cientistas $^{1}$. Julius Wagner-Jauregg (1857-1940), pela inoculação da malária (Plasmodium) para o tratamento da demência paralítica causada pela sífilis, Hideyo Noguchi (1876-1928), por seu trabalho sobre febre amarela e pela descoberta de que o Treponema pallidum é o agente causal da demência paralítica, e Émile Roux (ver acima) foram os que receberam maior número de indicações (nove, oito e oito, respectivamente). As indicações de Wagner-Jauregg e Noguchi foram avaliadas e recomendadas por membros do Comitê Nobel ou para tal designados (Hans C Jacobaeus, Bror Gadelius, Gunnar Hedrén, John Sjoqvist, Albin Dalén, Hilding Bergstrand, Carl Hesser e JE Johansson). As indicações de Roux não foram avaliadas e recomendadas. Wagner-Jauregg ganharia o prêmio Nobel de $1927^{28}$.

Uma possível explicação para a não concessão do prêmio Nobel em Fisiologia ou Medicina de 1921 e 1925 pode ser encontrada na pessoa do presidente do Comitê Nobel da época, Johan Erik Johansson, também conhecido como Jöns Johansson. Ele havia sido apresentado a Alfred Nobel no início da década de 1890 e trabalhado com transfusão de sangue no laboratório de Alfred Nobel, próximo de Paris ${ }^{23}$. Anos depois, JE Johansson se tornaria professor de Fisiologia no Instituto Karolinska e tomaria parte ativa na regulamentação dos estatutos da Fundação Nobel, particularmente para que a premiação em Fisiologia ou Medicina incluísse as ciências médicas clínicas e básicas. Posteriormente, 
ele seria indicado presidente do Comitê Nobel de Fisiologia ou Medicina para o período 1918-1926 ${ }^{19}$. JE Johansson era o único membro do Instituto Karolinska que havia trabalhado com Alfred Nobel e não havia ficado satisfeito com a escolha de alguns premiados pelo Nobel ${ }^{20}$. Ele efetivamente chegou a impedir a concessão do prêmio em alguns casos com a intenção de poupar recursos para construir um instituto de pesquisa Nobel. Esse instituto teria a tarefa de checar as novas descobertas anunciadas pelos indicados ao prêmio e assim auxiliar o Comitê Nobel no processo de seleção dos premiados. A coincidência do período de JE Johansson na presidência do Comitê Nobel (1918-1926) com a não concessão da premiação para Fisiologia ou Medicina nos anos de 1921 e 1925, além de sua insatisfação com a escolha de alguns premiados, sugere a participação ativa dele na tomada de decisões do Comitê Nobel. 0 papel proeminente que JE Johansson parece ter tido à frente do Comitê Nobel exemplifica o modo de atuação de muitas comissões avaliadoras, mesmo quando formadas pelos cientistas mais eminentes da instituição: nem todos os examinadores possuem o mesmo poder decisório, o mesmo prestígio; algumas opiniões às vezes contam mais do que as outras, ou influenciam mais ${ }^{22}$. Como se pode ver, esse elevado grau de hierarquia contribui para enfraquecer o sistema de avaliação por pares.

Em princípio, toda a atividade científica deveria ser avaliada pela qualidade dos problemas que coloca e investiga, e pela qualidade das soluções que oferecé ${ }^{4}$. A sequiência de descobertas conduzida por Carlos Chagas preenche integralmente esses critérios, pois ele descobriu uma nova doença (o problema) e ao identificar o agente etiológico, o modo de transmissão, 0 reservatório natural do parasita e a localização intradomiciliar do inseto transmissor, Carlos Chagas estava igualmente oferecendo possíveis caminhos para novas pesquisas visando soluções para erradicar o problema. 0 não reconhecimento das descobertas de Carlos Chagas, nessa segunda indicação ao prêmio Nobel, mostra mais uma vez que na prática a avaliação em ciência reflete principalmente as ligações que os membros da comunidade científica têm entre si, o que é considerado realização científica e 0 grau de hierarquia existente nas comissões avaliadoras. As ligações dos membros do Instituto Karolinska e do Comitê Nobel com a comunidade científica internacional, centrada quase que exclusivamente em cientistas europeus e norte-americanos, influenciavam nas escolhas dos indicadores, indicados e premiados, e sobre o que era considerada realização científica na época. Não há base para a hipótese de que a oposição enfrentada por Carlos Chagas por parte de alguns colegas em Manguinhos e na Academia Nacional de Medicina, que negavam a existência da doença ou tentavam reduzir sua importância, tenha sido um dos motivos para ele não ter recebido a premiação de 1921. Não há também documentos que comprovem a especulação de que o Comitê Nobel tenha se dirigido a instituições brasileiras e que essas tenham desaconselhado a premiação. 0 episódio da oposição a Carlos Chagas é profundamente lamentável, mas não deve ter influenciado a decisão quanto à premiação de 1921. As decisões do Comitê Nobel, como se tentou demonstrar neste texto, eram influenciadas por outros fatores.
A história da indicação de Carlos Chagas ao prêmio Nobel, da avaliação quase que informal de sua indicação e da sua não recomendação à premiação, é de grande valor para os historiadores da ciência, particularmente tendo em vista 0 conhecimento que se obteve sobre os fatores que condicionavam (e ainda condicionam) as escolhas de indicadores, indicados e ganhadores do prêmio, após a abertura e análise dos documentos dos arquivos do prêmio Nobel. Por fim, a premiação de Carlos Chagas com o Nobel, além fazer jus à sua genialidade e à relevância de suas descobertas, iria certamente dar maior visibilidade nacional e internacional à nova doença já nos primeiros anos após a sua descrição e ampliar o interesse pelo seu estudo, o que poderia resultar em grande benefício para a população afetada.

\section{REFERÊNCIAS}

1. Banco de dados de indicadores e indicados para o prêmio Nobel em Fisiologia ou Medicina, 1901-1951. Disponível em http://nobelprize.org/nomination/ medicine/database.html. Acesso em 06 de outubro de 2007.

2. Barré-Sinoussi F, Chermann J-C, Rey F, Nugeyre MT, Chamaret S, Gruest J, Dauguet C, Axler-Blin C, Vézinet-Brun F, Rouzioux C, Rozenbaum W, Montagnier L. Isolation of a T-lymphotropic retrovirus from a patient at risk for acquired immune deficiency syndrome (AIDS). Science 220: 868-871, 1983.

3. Biblioteca Virtual Carlos Chagas. Exposition of reasons for the presentation of Dr. Carlos Ribeiro Justiniano das Chagas as a candidate for the Nobel prize in Medicine. Rio de Janeiro: Manguinhos, 1920. Disponível em http://www4. prossiga.br/Chagas/sobrech/sec/tb-527.htm. Acesso em 18/11/2008.

4. Brenner S. The impact of society in science. Science 282: 1411-1412, 1998.

5. Chagas C. Neue Trypanosomen: vorläufige Mitteilung. Archiv für Schiffs-und Tropen-Hygiene 13: 120-122, 1909.

6. Chagas C. Nouvelle espèce de trypanosomiase humaine. Bulletin de La Société de Pathologie Exotique 2: 304-307, 1909.

7. Chagas C. Nova tripanozomiase humana: Estudos sobre a morfolojia e o ciclo evolutivo do Schizotrypanum cruzi n. gen., n. sp., ajente etiolojico de nova entidade morbida do homem. Memórias do Instituto Oswaldo Cruz 1: 159-218, 1909.

8. Chagas C. Über eine neue Trypanosomiasis des Menschen. Archiv für Schiffs- und Tropen-Hygiene 13: 351-353, 1909.

9. Chagas C. Le cycle de Schizotrypanum cruzi chez l'homme et les animaux de laboratoire. Bulletin de La Société de Pathologie Exotique 4: 467-471, 1911.

10. Chagas $C$. Nova entidade morbida do homem: rezumo geral de estudos etiolojicos e clinicos. Memórias do Instituto Oswaldo Cruz 3: 219-275, 1911.

11. Chagas C. Les formes nerveuses d'une nouvelle trypanosomiase (Trypanosoma cruzi inocule par Triatoma magista) (Maladie de Chagas). Nouvelle Iconographie de La Salpêtrière 26: 1-9, 1913.

12. Clavel F, Guétard D, Brun-Vézinet F, Chamaret S, Rey M-A, Santos-Ferreira MO, Laurent AG, Dauguet C, Katlama C, Rouzioux C, Klatzmann D, Champalimaud JL, Montagnier L. Isolation of a new human retrovirus from West African patients with AIDS. Science 233: 343-346, 1986.

13. Coutinho M. O Nobel perdido. Folha de São Paulo, 7 de fevereiro de 1999, Caderno 5, p. 11. Disponível em www.submarino.net/cchagas/artigos/art4.htm. Acesso em 18/11/2008.

14. Coutinho M, Freire Jr O, Dias JCP. The Noble enigma: Chagas' nomination for the Nobel prize. Memórias do Instituto Oswaldo Cruz 94 (Supl I): 123-129, 1999.

15. Crawford E. Nobel: always the winners, never the losers. Science 282: 1256-1257, 1998.

16. Dias JCP. Carlos Chagas: prêmio Nobel em 1921. Disponível em www.datasus. gov.br/cns/documentos/Chagas.htm. Acesso em 18/11/2008.

17. Dias JCP, Coura JR, Coutinho M. Carlos Chagas e a indicação ao prêmio Nobel. Disponível em www.fiocruz.br/chagas/cgi/cgilua.exe/sys/start.htm?sid=142. Acesso em 13/11/2008. 
18. Gouvêa HS. Trajetória profissional, Produção intelectual, Dados pessoais. Disponível em: www.dichhistoriasaude.coc.fiocruz.br/iah/P/verbetes/gouhilso. $\mathrm{htm}$. Acesso em 13/11/2008.

19. Karolinska Institutet. Kort historic. Stockholm, Sweden. Disponível em: http:// www.ki.se/essaer200ar/ki_historia.htm. Acesso em 16/11/2008.

20. Lindsten J, Ringertz N. The Nobel prize in Physiology or Medicine. In: Levinovitz WA, Ringertz N (ed) The Nobel prize: the first 100 years, Imperial College Press and World Scientific Publishing Co, Pte. Ltd, London, Singapore, p. 111-136, 2001. Disponível em: http://nobelprize.org/nobel_prizes/medicine/articles/lindstenringertz-ver/index.html. Acesso em 13/11/2008.

21. Merle M. 0 anticolonialismo. In: Ferro M (org) 0 livro negro do colonialismo, Ediouro, Rio de Janeiro, p. 708-747, 2004.

22. Mueller SPM. A comunicação científica e o movimento de acesso livre ao conhecimento. Ciência da Informação 35: 27-38, 2006.

23. Ringertz N. Alfred Nobel's health and his interest in medicine. Disponível em: http://nobelprize.org/cgi-bin/print?from=\%2Falfred_nobel\%2Fbiographical $\% 2$ Farticles\%2Fringertz\%2F. Acesso em 15/11/2008.
24. Scaravilli F. Tropical diseases : A historical perspective. Brain Pathology 7: 561$567,1997$.

25. Sierra-Iglesias JP. Salvador Mazza - su vida y su obra - redescubridor de la enfermedad de Chagas. Universidad Nacional de Jujuy, San Salvador de Jujuy, 1990.

26. The Nobel Foundation. Pessoas qualificadas para indicar candidatos ao Prêmio Nobel de Fisiologia ou Medicina. Disponível em: http://nobelprize. org/nomination/medicine/nominators.html. Acesso em 05/10/2008.

27. The Nobel Prize in Chemistry. Premiados com o Nobel de Química. Disponível em http://nobelprize.org/nobel_prizes/chemistry/laureates/. Acesso em 13/11/2008.

28. The Nobel Prize in Medicine. Premiados com o Nobel de Fisiologia ou Medicina Disponível em http://nobelprize.org/nobel_prizes/medicine/laureates/. Acesso em 13/11/2008.

29. The Nobel Prize in Physics. Premiados com o Nobel de Física. Disponível em http://nobelprize.org/nobel_prizes/physics/laureates. Acesso em 13/11/2008. 